\title{
A demonstration of auditory top-down processing
}

\author{
TERENCE HINES \\ Pace University, Pleasantville, New York
}

\begin{abstract}
A simple demonstration of auditory top-down processing is described in which one speech embedded in several others becomes much clearer when participants read a text of the target speech at the same time as they hear it.
\end{abstract}

Almost every introductory psychology textbook features some discussion of the constructive nature of perception, usually known as top-down processing. In topdown processing, an individual's knowledge and/or beliefs influence the way in which a stimulus is perceived. Such processing is vital to the veridical perception of the world. A creature would not long survive that could not in some way modify the information present-say, on the retinain order to make the retinal representation correlate more exactly with the nature of the real world. For example, the image on the retina changes size as an object moves toward or away from the viewer. It would be disastrous if an animal behaved as if objects in the real world changed size, instead of moving toward and away from it. Thus, size constancy, the phenomenon that as objects move toward or from us, they are perceived to move, but to retain a constant size, plays an important role in the survival of species that depend on vision for a major part of their guiding information in locomotion. The information that permits size constancy is stored in higher brain centers, although it is not yet known just where. Anatomically, this information interacts with and modifies, if that is what it actually does, the information arriving from the visual pathways.

Thus, overall, top-down processing modifies the incoming sensory signal in the light of information previously stored in such a way as to make the incoming signal more informative and useful. A particularly dramatic example of this is the illusion of the missing phoneme. Warren (1970) showed that if one replaced a single phoneme in a tape-recorded word with white noise, participants did not notice the substitution. In fact, participants clearly heard the phoneme, even if it is not present in the sensory signal. Higher brain centers have filled in the missing information; the result is the perception of an uninterrupted spoken word.

Warren and Warren (1970) showed that information occurring later in a sentence could alter how the missing phoneme was perceived. They presented participants with sentences like, "It was found that the _eel was on the

Correspondence should be addressed to T. Hines, Psychology Department, Pace University, Pleasantville, NY 10570-2799 (e-mail: thines@fsmail.pace.edu).
WORD." Here, the first phoneme of the sixth word was replaced with white noise. The final word of the sentence could be axle, orange, shoe, or table. The eel word the participants heard in the sentence depended strongly on which word was at the end of the sentence. Thus, if the final word was orange, the white noise burst was perceptually replaced by $p$ to make peel. If the last word was shoe, participants heard heel, and so forth.

In the vast majority of cases, top-down processing is beneficial in that it brings the incoming sensory information more closely into correspondence with the real world the organism is faced with. It must be pointed out, however, that in certain cases, especially where the incoming sensory input is minimal, top-down processing can result in misperceptions. This is especially the case where observers hold mistaken beliefs or expectations about the nature of the world. This is probably most dramatically demonstrated by the tendency of witnesses to misperceive essentially random patterns of aircraft lights in the night sky as flying saucers (see Hines, 1988, for details).

Different introductory texts use different examples of top-down processing, but, by the nature of the printed text, these tend to be visual illusions of various varieties. Although these are of interest, no matter how well done, visual examples cannot provide a demonstration in the auditory mode. This report describes an auditory example of top-down processing that is both easy to do in the classroom setting and extremely compelling.

\section{METHOD}

\section{Materials}

A segment of the 1972 film Sensory World, currently available for $\$ 97$ from CRM Films (2215 Faraday Avenue, Carlsbad, CA 92008) in videotape format, served as the stimulus for this demonstration. The specific segment was intended by the producers of the film as a demonstration of auditory masking. In the segment, several different male voices are heard, speaking at the same time. One of those voices is that of Richard $M$. Nixon, delivering the first portion of his June 17,1970 speech on the nation's economy. Nixon is only heard during this segment-he is not visually present on the screen. The screen is filled with an unintelligible jumble of printed words. The portion of the speech heard is reprinted in the Appendix.

\section{Participants}

Students in two different introductory psychology classes served as participants in the present demonstration. There were 21 students 
in the author's Psychology 104 (Introductory Psychology 1) and 18 in his Psychology 105 (Introductory Psychology 2) class.

\section{Procedure}

Participants in both classes watched the Sensory World tape. Just before the segment on masking started, the tape was paused. The instructor then described what would be heard and asked the students in both classes to attempt to listen selectively to Nixon's voice and make a judgment on how easy it was to do so. They were asked to rate the ease of selectively listening to Nixon on a scale of 1 (voice impossible to distinguish from the background) to 10 (voice perfectly clear) after hearing the entire segment

At the end of the segment, and after participants had completed their initial rating, the tape was rewound to the beginning of the masking segment. Students in Psychology 105 then heard the segment again and were asked to rate the difficulty of listening selectively to Nixon the second time through. Students in Psychology 104 were given a printed text of Nixon's speech (reproduced in the Appendix) and told to read the text as they listened to the segment a second time. They, too, then rated the difficulty a second time.

\section{RESULTS}

The results are shown in Table 1 . There was a significant main effect of class $[F(1,37)=36.64, p<.001]$ and a significant main effect of presentation [first vs. second, $F(1,17)=21.64, p<.001]$. Most importantly, there was a significant interaction between these two variables $[F(1,17)=6.42, p<.025]$. Inspection of Table 1 suggests that this interaction is due to the very large increase in the rated clarity of Nixon's voice when participants in Psychology 104 heard the segment the second time, while reading the speech. This is confirmed through a post hoc Scheffé test, which showed that the critical difference between means at the .05 level was 2.68 . Given this, the difference between Psychology 104's rating with and without the printed text is significant. The tiny improvement seen in Psychology 105 from the first to the second hearing of the segment without the printed text did not reach significance.

\section{DISCUSSION}

The dramatic increase in the rated clarity of Nixon's speech when students read the text constitutes an impressive demonstration of top-down processing. Obviously, the pattern of sound waves that struck their ears during the two presentations of the masking segment had not changed from the first to the second presentation. What had changed was their expectations. And these expectations, derived from the printed text, operated to render the passage in
Table 1

Clarity Ratings (Means and Standard Deviations) for the Two Classes for the First and Second Hearings

\begin{tabular}{cccccc}
\hline & \multicolumn{2}{c}{ First Hearing } & & \multicolumn{2}{c}{ Second Hearing } \\
\cline { 2 - 3 } \cline { 5 - 6 } Group & $M$ & $S D$ & & $M$ & $S D$ \\
\hline Psychology 104 & 4.1 & 2.3 & & 8.7 & 1.6 \\
Psychology 105 & 3.3 & 2.2 & & 3.7 & 2.2 \\
\hline
\end{tabular}

question much easier to perceive. Students find this a most impressive demonstration, as, indeed, it is.

It must be pointed out that the students in Psychology 105 were more advanced than those in Psychology 104. None in Psychology 105, however, had heard the demonstration in their previous Psychology 104 class. It seems extremely unlikely that the obtained result was due to differences in knowledge of the field on the part the students in the more advanced course. In the interest of complete reporting, it should be noted that the Psychology 105 students heard the segment a third time, this time with the printed text. On this third time through, they rated the clarity at 8.4. This is not significantly different from the rating of the Psychology 104 group (8.7) when they heard the speech while reading the text $[t(37)=.35]$.

\section{REFERENCES}

HINES, T. (1988). Pseudoscience and the paranormal: A critical examination of the evidence. Buffalo, NY: Prometheus Books.

WARREN, R. (1970). Perceptual restoration of missing speech sounds. Science, 167, 392-393.

WARREN, R., \& WARREN, R. (1970). Auditory illusions and confusions. Scientific American, 223(6), 30-36.

\section{APPENDIX \\ Portion of President Nixon's June 17, 1970 Speech on the Economy Heard in the Masking Segment in Sensory World}

Good afternoon my fellow Americans:

Today I would like to share with you my thoughts on three subjects that reach into the homes and the pocketbooks of every family: your job, your income, and your cost of living.

Specifically, I shall announce actions that will help to move us ahead more quickly towards our goal of full employment, economic growth, and reasonable price stability in peacetime.

Let us begin by recognizing these facts:

The American economy is the strongest in the world. This year, the number of Americans who have jobs is the highest in our history.

(Manuscript received August 11, 1997; revision accepted for publication April 10, 1998.) 